\section{Case Report}

Korean J Transplant 2021;35:183-188 https://doi.org/10.4285/kjt.20.0059
Received December 22, 2020

Revised March 7, 2021

Accepted March 31, 2021

Corresponding author: Shin Hwang Department of Surgery, Asan Medical Center, University of Ulsan College of Medicine, 88 Olympic-ro 43-gil, Songpagu, Seoul 05505, Korea

Tel: +82-2-3010-3930

Fax: +82-2-3010-6701

E-mail: shwang@amc.seoul.kr

(C) The Korean Society for Transplantation This is an Open Access article distributed under the terms of the Creative Commons Attribution Non-Commercial License (http://creativecommons.org/licenses/ by-nc/4.0/) which permits unrestricted non-commercial use, distribution, and reproduction in any medium, provided the original work is properly cited.

\section{$\mathrm{KJT}^{\mathrm{K}}$ \\ KOREAN JOURNAL OF TRANSPLANTATION}

pISSN 2671-8790

elSSN 2671-8804

\title{
Hepatic artery reconstruction using interposition of autologous saphenous vein conduit for living donor liver transplantation: a case report
}

\author{
Deok-Bog Moon, Shin Hwang, Dong-Hwan Jung, Chul-Soo Ahn, Gil-Chun Park, \\ Tae-Yong Ha, Gi-Won Song, Young-In Yoon, Sung-Gyu Lee
}

Division of Hepatobiliary Surgery and Liver Transplantation, Department of Surgery, Asan Medical Center, University of Ulsan College of Medicine, Seoul, Korea

We have preferentially used the right gastroepiploic artery (RGEA) as an alternative for the recipient hepatic artery $(\mathrm{HA})$ inflow during living donor liver transplantation (LDLT), but it was not always available. We herein present a case of adult LDLT with HA reconstruction using a greater saphenous vein (GSV) conduit because of the absence of the RGEA due to prior subtotal gastrectomy. A 55-year-old male patient diagnosed with hepatitis $B$ virus-associated liver cirrhosis and secondary biliary cirrhosis underwent LDLT using a modified right liver graft. The upper abdominal cavity was heavily adhered due to prior abdominal surgeries, thus we had to sacrifice the common bile duct and the right HA completely. A 6-cm-long GSV segment was harvested from the left ankle and interposed between the recipient gastroduodenal artery and the graft HA. The patient recovered from LDLT and HA complications did not occur. However, 8 years after LDLT, chronic rejection occurred, thus repeated deceased donor liver transplantation was performed. This patient has been doing well for 2 years after retransplantation. In conclusion, we suggest that interposition of an autologous GSV conduit can be an alternative for establishing HA inflow in LDLT when other inflow source is not available.

Keywords: Liver transplantation; Hepatic artery thrombosis; Vascular interposition; Living donor; Saphenous vein; Case report

\section{INTRODUCTION}

Hepatic arterial blood supply is essential for successful liver transplantation. Unlike deceased donor liver transplantation (DDLT), special attention is required during living donor liver transplantation (LDLT) to secure the sources of hepatic artery (HA) inflow because a jump graft from the aorta is usually not feasible. However, meticulous dissection of the hilar structures to avoid iatrogenic HA injury does not always ensure obtainment of an inflow HA branch because of previous $\mathrm{HA}$ injuries from multiple episodes of transarterial chemoembolization, atherosclerosis, scarring from the previous liver surgery, or other factors. When a native HA branch was not available, we have preferentially used the right gastroepiploic artery (RGEA) because of its invariable anatomic location, adequate size matching, and long length [1]. However, the RGEA was not always not available, especially in patients who had previously undergone upper 


\section{HIGHLIGHTS}

-We present a case of adult living donor liver transplantation with hepatic artery reconstruction using a greater saphenous vein conduit.

- Interposition of an autologous greater saphenous vein conduit can be an alternative for establishing hepatic artery inflow in living donor liver transplantation when other inflow source is not available.

abdominal surgery. In such a difficult situation, another source of inflow artery should be identified. We herein present a case of adult LDLT with HA reconstruction using interposition of a greater saphenous vein (GSV) conduit.

\section{CASE REPORT}

This study was approved by the Institutional Review Board of Asan Medical Center (IRB No. 2020-0836), and the requirement for informed consent was waived.

A 55-year-old male patient diagnosed with hepatitis $B$ virus (HBV)-associated liver cirrhosis was admitted to our institution under the impression of hepatic encephalopathy. This patient had undergone subtotal gastrectomy and gastrojejunostomy due to duodenal ulcer 24 years ago, and left lateral sectionectomy due to hepatolithiasis 11 years ago. Four years ago, surgical treatment was attempted for recurrent left intrahepatic duct stones, but only T-tube cholangiolithotomy was performed due to heavy abdominal adhesion. He was also diagnosed with ankylosing spondylitis and autoimmune cholangitis requiring steroid medication and percutaneous transhepatic biliary drainage. This patient was finally diagnosed with HBV-associated liver cirrhosis combined with secondary biliary cirrhosis (Fig. 1). The model for end-stage liver disease (MELD) score was 22. This patient was classified into the Korean Network for Organ Sharing (KONOS) old category 2B. Because there was a low possibility of being allocated to DDLT, LDLT was performed because the general condition of the patient was gradually deteriorating.

The donor was the 26-year-old daughter of the patient. The modified right liver graft from this donor weighed $570 \mathrm{~g}$, making a graft-to-recipient weight ratio of $1.19 \%$. The donor recovered uneventfully from the donor operation and was discharged 8 days after the operation. During the recipient operation, the upper abdominal cavity was heavily adhered due to previous surgeries. Because of very heavy adhesion and scarring at the hepatoduodenal ligament, it was not feasible to dissect the right HA securely. We had to transect the common bile duct and the right HA completely. Recipient hepatectomy was continued according to the standard procedure of LDLT.

Because the RGEA was not available due to prior subtotal gastrectomy, we decided to perform HA reconstruction with interposition of an autologous vessel conduit interposition between the graft HA and the recipient gastroduodenal artery. Thus, the GSV in the left ankle was dissected, and a 6-cm-long GSV segment was harvested through the conventional open method.

At the back table, the right hepatic vein orifice of the liver graft was enlarged with incision and patch venoplasty. The middle hepatic vein orifices at the graft liver cut surface were reconstructed with a cryopreserved iliac vein conduit. Immediately after finishing the bench work for the liver graft, the modified right liver graft was implanted according to the standard procedure of LDLT. The graft right hepatic vein and the interposed middle hepatic vein conduit were separately reconstructed to the recipient's
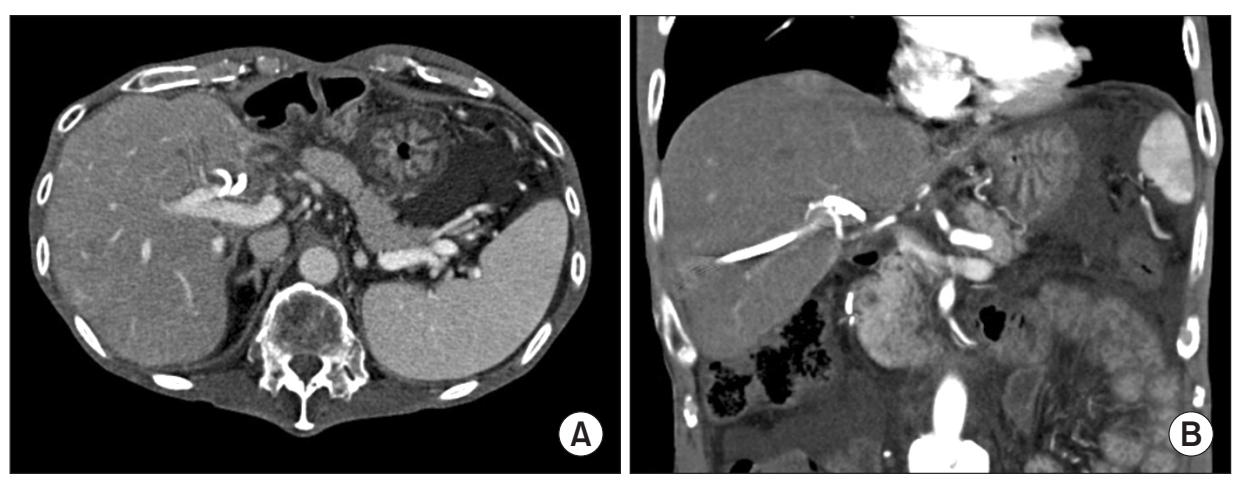

Fig. 1. Pretransplant dynamic computed tomography findings showing percutaneous transhepatic biliary drainage tube $(A)$ and visualization of the right hepatic artery (B). 
right hepatic vein and left-middle hepatic vein stumps, respectively. The recipient portal bifurcation was used for anastomosis with the graft portal vein (Fig. 2).

The recipient gastroduodenal artery was meticulously dissected and both the proximal and distal sides were securely clamped. The distal-side end of the GSV segment was anastomosed with gastroduodenal artery under surgical microscopy. After brisk arterial blood flow was identified, the proximal-side end of the GSV segment was anastomosed with the graft HA stump (Fig. 3). Biliary reconstruction was performed with Roux-en-Y hepaticojejunostomy. The skin-to-skin total operation time was 14 hours and 31 units of red blood cells were infused during the operation because of heavy abdominal adhesion and

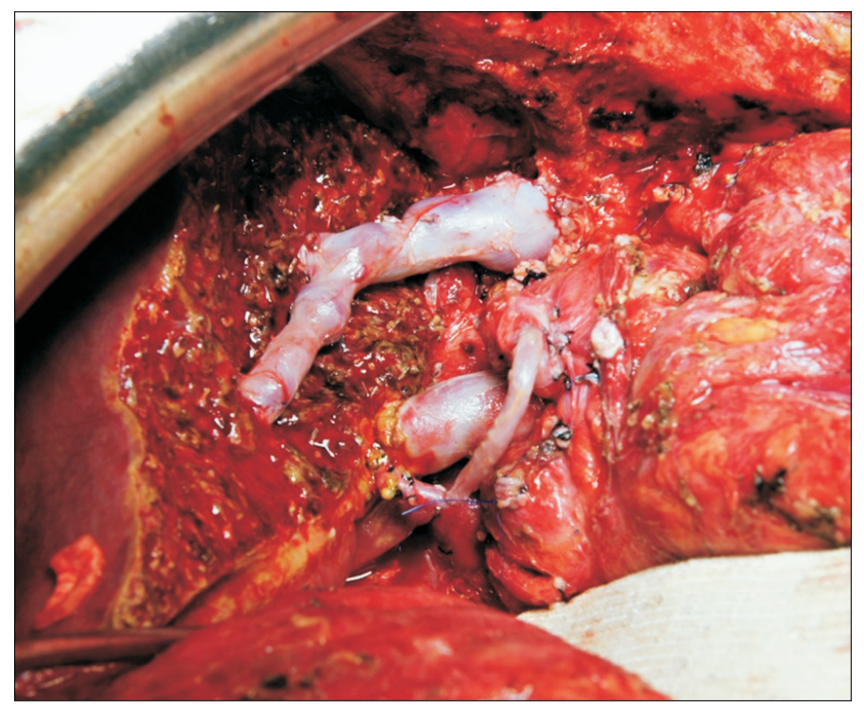

Fig. 2. Intraoperative photograph showing completion of vascular reconstruction of a modified right liver graft.

\section{GSV interposition.}

The pathology report of the explant liver showed that there was secondary biliary cirrhosis possibly associated with hepatolithiasis and chronic cholangitis with extensive hepatocyte necrosis (Fig. 4). The patient recovered slowly from the LDLT operation (Fig. 5) and was finally discharged from the hospital at 59 days after transplantation. This patient experienced acute rejection (rejection activity index, 4) at 4 months after LDLT, which was well controlled with steroid therapy.

However, 8 years after LDLT, progressive aggravation of jaundice occurred, in which the liver biopsy finding was compatible with chronic rejection (Fig. 6A and B). This patient was enlisted at the KONOS waitlist with an initial MELD score of 28. After waiting for 2 months, at the updated MELD score of 35 , he was allocated to receive

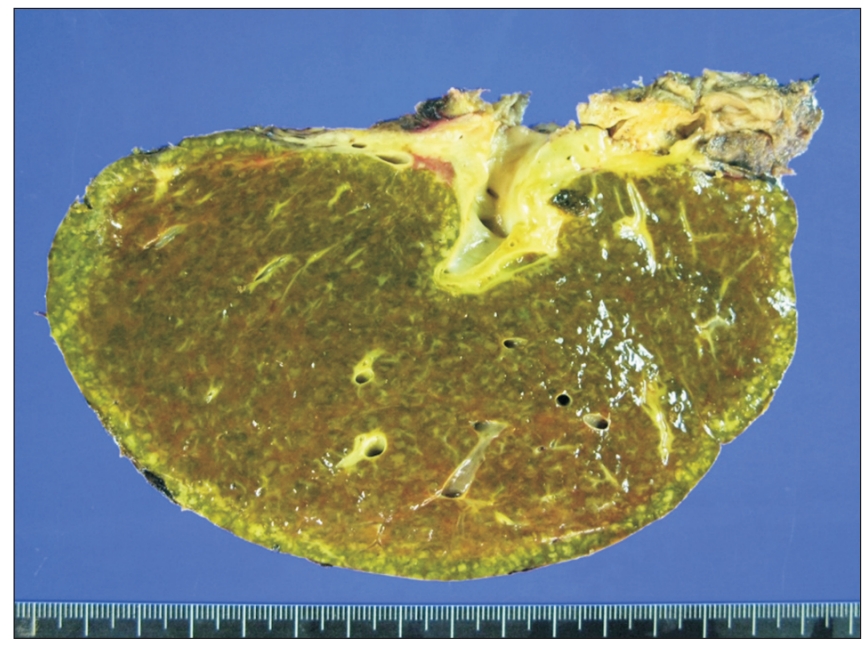

Fig. 4. Photographs of the explant liver showing secondary biliary cirrhosis associated with hepatolithiasis and chronic cholangitis.
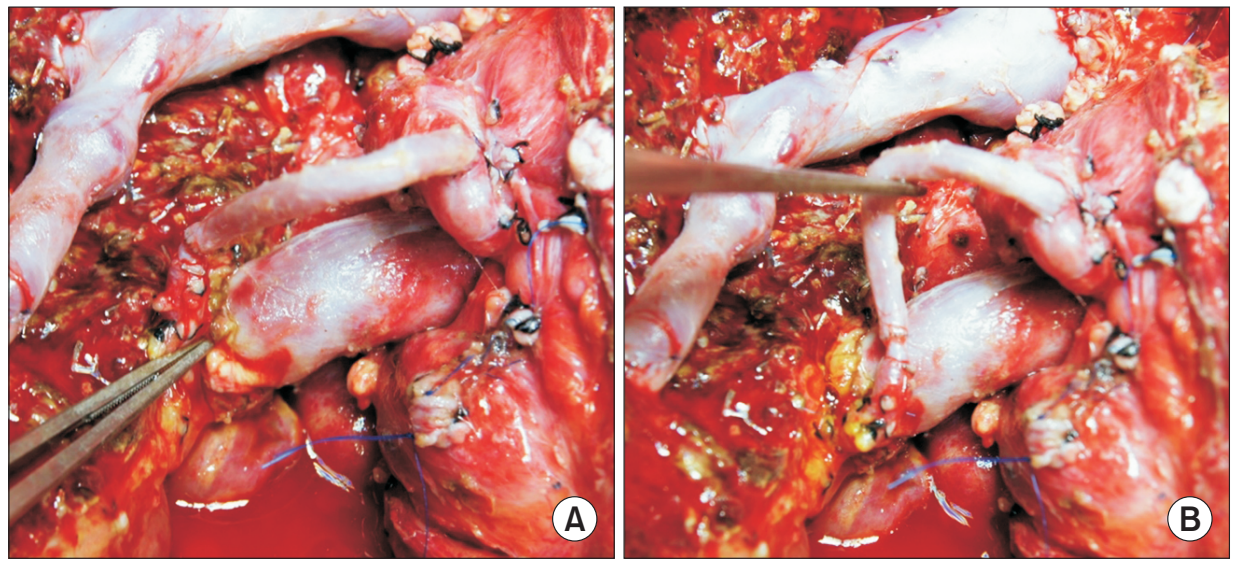

Fig. 3. Intraoperative photographs showing interposition of the greater saphenous vein conduit between the recipient gastroduodenal artery $(A)$ and the graft right hepatic artery (B). 

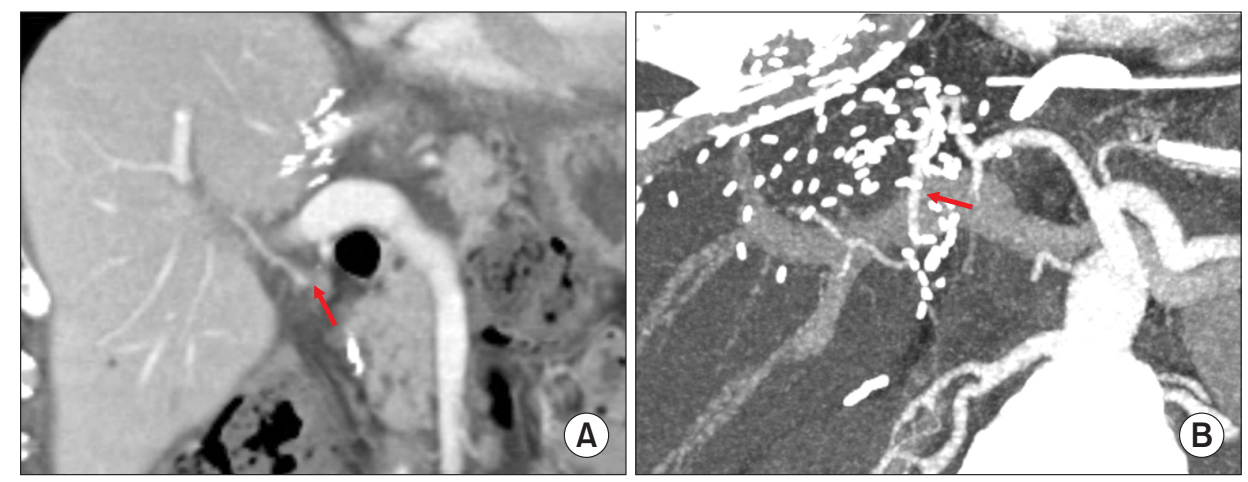

Fig. 5. Posttransplant liver dynamic computed tomography findings taken at 2 weeks after transplantation. Uneventful visualization of the portal vein $(A)$ and the right hepatic artery (B) is observed. Arrows indicate the running course of the greater saphenous vein conduit.
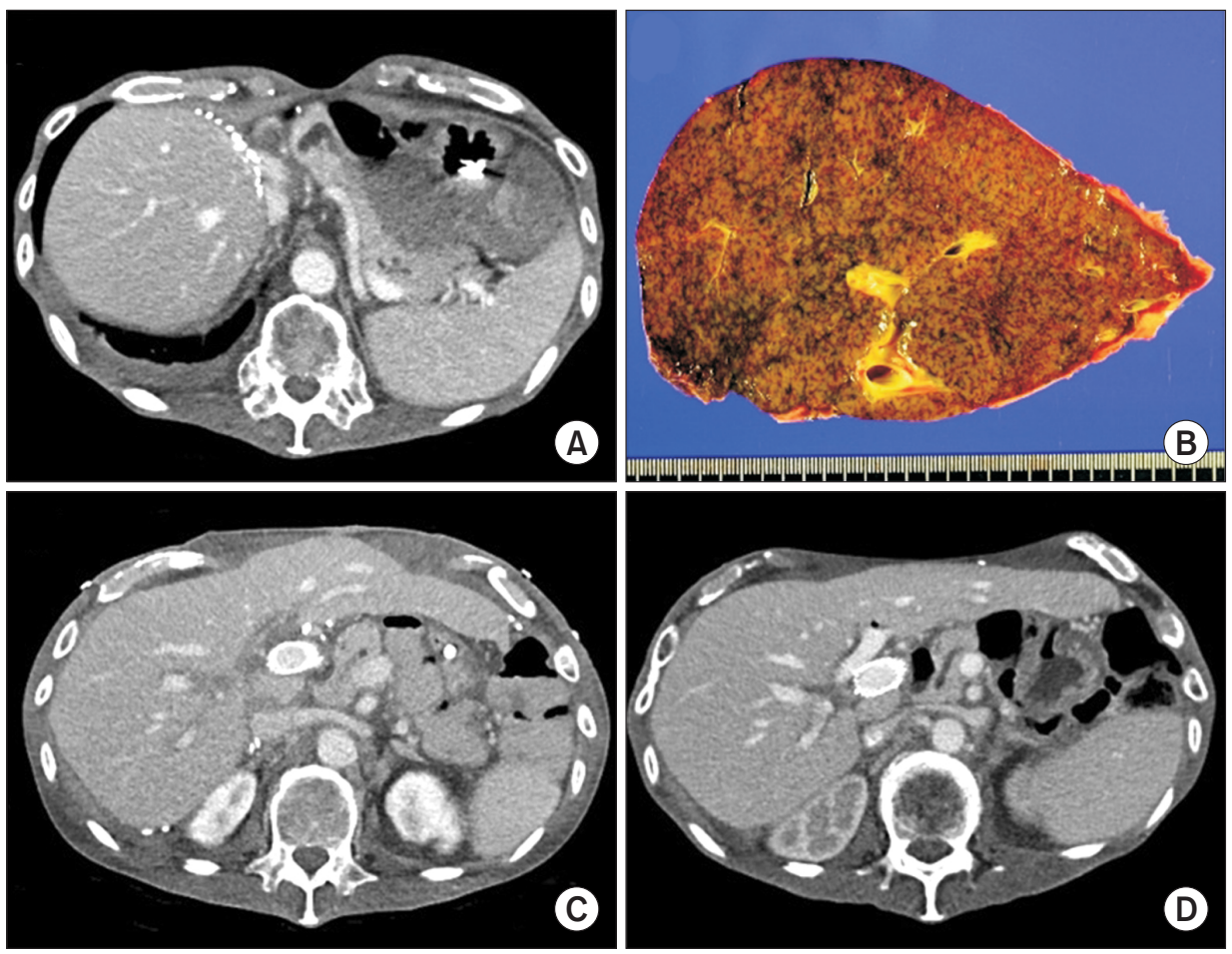

Fig. 6. Posttransplant findings around retransplantation. Computed tomography (CT) scan taken at 8 years after living donor liver transplantation shows liver cirrhosis with splenomegaly (A). The explant liver shows typical findings of chronic rejection (B). No abnormal finding is observed in the CT images taken 3 weeks (C) and 1 year (D) after deceased donor liver retransplantation except for portal vein stenting.

DDLT with a whole liver graft from a 52-year-old marginal donor. The standard procedure of DDLT with inferior vena cava interposition was performed and the graft HA was anastomosed with the recipient common HA after removal of the previously interposed GSV conduit. The portal vein reconstruction was patent, but significant angulation existed, thus intraoperative portal vein stenting was performed (Fig. 6C). At 40 days after retransplantation, acute rejection (rejection activity index, 4) developed. The patient recovered slowly from retransplantation due to early graft dysfunction and pulmonary complications, and he was finally discharged from the hospital at 151 days after retransplantation. This patient has been doing well for 2 years after retransplantation (Fig. 6D).

\section{DISCUSSION}

The use of a GSV conduit for HA reconstruction has been rarely practiced during LDLT because the native recipient HAs can be used in a majority of patients and other extra-anatomical arteries, such as RGEA, right gastric artery, left gastric artery, splenic artery, mid colic artery, jejunal artery and other arteries, can be an alternative [1-3].

According to our experience on redo HA reconstruc- 
tion in 21 cases of LDLT suffering from HA thrombosis, the sources of arterial inflow were the RGEA in 15 patients, previous native HA in three patients, and interposition graft from the aorta in one patient [2]. A study from Kyushu University revealed that 33 extra-anatomical HA reconstructions were performed in 22 patients with primary LDLT (6.6\%), in four patients with re-LDLT (50\%), and in four patients with HA re-reconstructions for HA complications $(80 \%)$ [3]. The recipient inflow arteries were the RGEA in 12 patients, right gastric artery in six patients, gastroduodenal artery in five patients, left gastric artery in two patients, splenic artery in two patients, cystic artery in two patients, and interposition grafts (superior rectal artery in one patient, right gastric vein in one patient, and splenic artery in two patients) in four patients. Only one case of HA-related complication, the formation of an aneurysm, was found after extra-anatomical HA reconstruction. Considering these data, the use of a GSV conduit was an exceptional solution in our current setting of LDLT because the RGEA and other extra-anatomical arteries were not available in the present case.

Unlike the rarity of a GSV conduit in our experience, a study from Pakistan reported 21 cases of LDLT using a GSV conduit, in which HA thrombosis occurred in only one patient $(4.7 \%)$ [4]. This result was comparable to that in 452 cases using the native recipient HAs. The fact that GSV conduits were used in $4.4 \%$ of the LDLT cases indicates that the authors have preferentially used GSV conduits instead of the RGEA. We think that such a preference for GSV conduits does not appear reasonable although they have achieved good outcomes.

The GSV conduit is different from the other types of arterial inflow sources because it is an interposition jump graft requiring two anastomoses as in coronary artery bypass grafting (CABG) surgery [5]. The GSV is a smallsized thick-walled vein, thus it has been frequently used as an arterial substitute for CABG for a long period [6]. Very long patency of patent CABG with a GSV graft has been reported [7], but there was a potential risk of aneurysm and pseudoaneurysm after the CABG using GSV grafts $[8,9]$. The present case underwent retransplantation due to chronic rejection 8 years after LDLT, in which the GSV conduit was patent without any vascular complications throughout the 8-year period.

The available length of GSV conduits can be increased to more than $20 \mathrm{~cm}$, which enables performing a long extra-anatomic jump graft. There are case reports presenting successful application of a supraceliac aorto-hepatic conduit and a right iliac artery-hepatic conduit using a GSV graft during LDLT $[10,11]$. To protect the GSV graft from potential harvest-associated injury, no-touch technique is preferred. A prospective, randomized study with 156 patients who underwent CABG compared three GSV harvesting techniques: conventional, intermediate, and no-touch [12]. In the conventional technique, the GSV was harvested through a longitudinal incision in the leg. The adventitial layer was removed, and tributaries were ligated with sutures. The vein was removed immediately after dissection, distended with saline solution injected with a syringe at a constant pressure of $300 \mathrm{mmHg}$ for 1 minute, and then stored in saline solution at room temperature. In the intermediate technique, the GSV was dissected along the conventional technique, but was not immediately distended. It was left in situ and covered with a compress soaked in saline solution containing papaverine, and perfusion was maintained. After removal, the GSV was stored in blood obtained from the cannula introduced in the aorta. Although papaverine was used, all veins had to be distended because of spasm. In the notouch technique, a continuous incision in the leg exposed the saphenous vein. All visible tributaries were ligated at about $0.5 \mathrm{~cm}$ from the GSV wall with sutures. The GSV and a surrounding tissue pedicle were isolated and left in situ; perfusion was maintained, and the vein was covered with a compress soaked in pure saline solution. After removal from the leg, the GSV was stored in blood obtained from the arterial cannula. This study presented that the preservation of the endothelial cell integrity was greater in the no-touch technique than in the other procedures. At angiographic follow-up, the patency for the no-touch group was $95.4 \%, 88.9 \%$ for the grafts of the conventional technique group, and $86.2 \%$ for the grafts performed in the intermediate technique group. The immunohistochemical assessment revealed endothelial enzyme nitric oxide synthase in all three layers of the vein wall in the no-touch group and reduction of this enzyme in the conventional group [12].

Endoscopic GSV harvesting has been also reported because it can reduce the leg wound complications [13-15]. However, for LDLT, we have used the conventional open harvesting method because GSV harvesting is a small part in the whole surgical procedures of LDLT and the endoscopic method requires more time and effort. In conclusion, we think that interposition of an autologous GSV conduit can be an alternative for establishing HA inflow in LDLT when other inflow source is not available. 


\section{ACKNOWLEDGMENTS}

\section{Conflict of Interest}

No potential conflict of interest relevant to this article was reported.

\section{Funding/Support}

This study was supported by research grant from the Korean Society for Transplantation (2021-00-03006-018).

\section{ORCID}

Deok-Bog Moon

Shin Hwang

Dong-Hwan Jung

Chul-Soo Ahn

Gil-Chun Park

Tae-Yong $\mathrm{Ha}$

Gi-Won Song

Young-In Yoon

Sung-Gyu Lee

https://orcid.org/0000-0002-8209-3540 https://orcid.org/0000-0002-9045-2531 https://orcid.org/0000-0001-5984-023X https://orcid.org/0000-0002-3844-3646 https://orcid.org/0000-0003-1631-3258 https://orcid.org/0000-0001-9932-0212 https://orcid.org/0000-0002-4235-0434 https://orcid.org/0000-0002-9308-0366 https://orcid.org/0000-0001-9161-3491

\section{Author Contributions}

Conceptualization: SH. Data curation: DBM, DHJ, CSA, GCP. Formal analysis: TYH. Methodology: GWS, YIY, SGL. Project administration \& Visualization: $\mathrm{SH}$. Writing-original draft: DBM, SH. Writing-review \& editing: SH.

\section{REFERENCES}

1. Ahn CS, Hwang S, Moon DB, Song GW, Ha TY, Park GC, et al. Right gastroepiploic artery is the first alternative inflow source for hepatic arterial reconstruction in living donor liver transplantation. Transplant Proc 2012;44:451-3.

2. Park GC, Moon DB, Kang SH, Ahn CS, Hwang S, Kim $\mathrm{KH}$, et al. Overcoming hepatic artery thrombosis after living donor liver transplantations: an experience from Asan Medical Center. Ann Transplant 2019;24:588-93.

3. Uchiyama H, Shirabe K, Taketomi A, Soejima Y, Ninomiya $\mathrm{M}$, Kayashima $\mathrm{H}$, et al. Extra-anatomical hepatic artery reconstruction in living donor liver transplantation: can this procedure save hepatic grafts? Liver Transpl 2010;16:1054-61.

4. Bhatti AB, Dar FS, Qureshi Al, Haider S, Khan NA. Saphenous vein conduits for hepatic arterial reconstruction in living donor liver transplantation. Langenbecks
Arch Surg 2019;404:293-300.

5. Tranbaugh RF, Schwann TA, Swistel DG, Dimitrova KR, Al-Shaar L, Hoffman DM, et al. Coronary artery bypass graft surgery using the radial artery, right internal thoracic artery, or saphenous vein as the second conduit. Ann Thorac Surg 2017;104:553-9.

6. Gaudino M, Taggart D, Suma H, Puskas JD, Crea F, Massetti $\mathrm{M}$. The choice of conduits in coronary artery bypass surgery. J Am Coll Cardiol 2015;66:1729-37.

7. Furukawa $\mathrm{H}$, Yamane $\mathrm{N}$, Honda $\mathrm{T}$, Yamasawa T, Kanaoka Y, Tanemoto K. Angiographic appearance of patent saphenous vein graft 32 years after coronary artery bypass grafting. Circ J 2019;83:840.

8. Queiroz RM, Nastri R Filho, Ferez MA, Costa MJ, Laguna CB, Valentin MV. Thrombosed aneurysm of saphenous vein coronary artery bypass grafting. Rev Assoc Med Bras (1992) 2017;63:488-91.

9. Dubois CL, Vandervoort PM. Aneurysms and pseudoaneurysms of coronary arteries and saphenous vein coronary artery bypass grafts: a case report and literature review. Acta Cardiol 2001;56:263-7.

10. Li PC, Thorat A, Jeng LB, Yang HR, Li ML, Yeh CC, et al. Successful application of supraceliac aortohepatic conduit using saphenous venous graft in right Lobe living donor liver transplantation. Liver Transpl 2017;23:976-80.

11. Baimakhanov Z, Magauina A, Matkerimov A, Kaniev S, Doskhanov M, Serikuly E, et al. Extra-anatomic jump graft arterial reconstruction using a great saphenous vein autograft during living donor liver transplantation. Transplant Proc 2019;51:3120-3.

12. de Rueda F, Souza D, Lima Rde C, Menezes A, Johansson $B$, Dashwood $M$, et al. Novel no-touch technique of harvesting the saphenous vein for coronary artery bypass grafting. Arq Bras Cardiol 2008;90:356-62.

13. Kan CD, Luo CY, Yang YJ. Endoscopic saphenous vein harvest decreases leg wound complication in coronary artery bypass grafting patients. J Card Surg 1999;14:157-62.

14. Felisky CD, Paull DL, Hill ME, Hall RA, Ditkoff M, Campbell WG, et al. Endoscopic greater saphenous vein harvesting reduces the morbidity of coronary artery bypass surgery. Am J Surg 2002;183:576-9.

15. Bitondo JM, Daggett WM, Torchiana DF, Akins CW, Hilgenberg AD, Vlahakes $G J$, et al. Endoscopic versus open saphenous vein harvest: a comparison of postoperative wound complications. Ann Thorac Surg 2002;73:523-8. 